\title{
Das Projekt ATHENE - Ausgründung von technologieorientierten Unternehmen aus Hochschulen und außeruniversitären Forschungseinrichtungen
}

\author{
Eberhard Brink, Bernd Groß
}

\section{Das Projekł ATHENE}

(Ausgründung von Technologieunternehmen aus HochschulEinrichtungen und Naturwissenschaflich-technischen Einrichtungen)

Das Bundesministerium für Bildung, Wissenschaft, Forschung und Technologie (BMBF) beauftragte im Herbst 1995 vier Einrichtungen mit der Durchführung des Projektes ATHENE, das die Erfassung und Stimulierung von Unternehmensausgründungen aus Hochschulen und außeruniversitären Forschungseinrichtungen zum Inhalt hat. Mit Kofinanzierungen wurde das Projekt durch die Länder Berlin, Niedersachsen, Nordrhein-Westfalen, Sachsen und Schleswig-Holstein sowie durch die Deutsche Ausgleichsbank (DtA), den Deutschen Sparkassenund Giroverband (DSGV), den Gerling Konzern und die Deutsche Bank unterstützt.

Ziel des Projektes war es, den Stand und die Perspektiven akademischer Unternehmensgründungen in Deutschland zu erfassen, das Gründungsverhalten von Mitarbeitern von Hochschulen und außeruniversitären Forschungseinrichtungen zu analysieren, akademische Unternehmensgründungen zu charakterisieren, Potentiale und Entwicklungstrends zu bestimmen, hemmende und fördernde Faktoren herauszuarbeiten und erfolgreiche Herangehensweisen (Best Practice) aufzuzeigen. Zu diesem Zweck wurden durch die Projektpartner in den verschiedenen Inkubationseinrichtungen (Hochschulen, Forschungsinstituten, Technologie- und Gründerzentren) Erhebungen und Interwievs sowohl unter den Leitern solcher Einrichtungen als auch den potentiellen Gründern und unter den gegründeten Unternehmen durchgeführt.

Mit dem Projekt soll ein Beitrag zur signifikanten Erhöhung der Zahl von Unternehmensneugründungen aus Wissenschaftseinrichtungen geleistet werden, um auf diesem Wege den Prozeß der Überführung wissenschaftlicher Forschungsergebnisse in marktfähige Produkte zu beschleunigen.

\section{Deutschland auf dem Weg zu mehr Selbständigkeit und Unternehmertum}

Innovation ist auch ein Motor der sozialen Entwicklung. Ohne eine ständige Erneuerung der technologischen Basis ist eine gesunde wirtschaftliche Entwicklung, die die wichtigste Grundlage sozialen Wohlstandes bildet, nicht zu erreichen. Vor dieser Herausforderung stehen alle entwickelten Industriestaaten, die heute mit fundamentalen Umbrïichen in ihrer Industriestruktur und mit einer zunehmenden Arbeitslosigkeit konfrontiert sind. Die Beherrschung der Innovation als belebender Faktor der Wirtschaft beinhaltet vor allem die schnellere Umsetzung neuester Forschungsergebnisse in marktfähige technologische Produkte und innovative Dienstleistungen. Das Fundament bilden eine moderne Bildung und eine leistungsfähige Forschung. Deren Umsetzung in marktfähige Produkte und Dienstleistungen ist heute immer stärker mit der Förderung kleiner und mittelständischer innovativer Unternehmen verbunden.

Beginnend in den 50er Jahren gründeten in den USA verstärkt Studenten und Absolventen kleine Unternehmen. Ihre Pionierrolle in der Entwicklung der Mikroelektronik und Computertechnik ist unbestritten. Silicon Valley wurde zu einem Symbol für eine Generation akademischer Unternehmer.

Die Bestimmung der Rolle kleiner innovativer Unternehmen nimmt einen Schwerpunkt in den aktuellen Diskussionen zur Wirtschaftsstrategie in den OECD-Staaten ein. Dabei geht es nicht nur um deren Anteil an den Arbeitsplätzen in der gesamten Wirtschaft, sondern um deren Bedeutung für die Herausbildung neuer Industrien mit dynamischen Wachstumspotentialen. Eine Gewichtung allein nach den in diesen Unternehmen geschaffenen Arbeitsplätzen wird den sich aus der Wirtschaftsdynamik ergebenden Anforderungen nicht gerecht. Zudem verdeutlichen die heute sichtbaren Umstrukturierungen in der Wirtschaft, daß in mittelständischen Unternehmen - nach Angaben von Creditreform - in einer gleichen Größenordnung von ca. 1 Mio. neue Arbeitsplätze geschaffen werden, wie diese in großen Wirtschaftsunternehmen abgebaut werden.

Die Gründung innovativer Unternehmen vollzieht sich heute zunehmend in Form von Spin-off-Gründungen durch Akademiker. Akademische Unternehmensgründungen als Quelle der Schaffung von Technologieunternehmen und innovativen Dienstleistungsunternehmen lassen sich damit auch nicht auf jene Unternehmensgründungen beschränken, die direkt aus Hochschulen und außeruniversitären Forschungseinrichtungen heraus vollzogen werden. Oft erfolgen Gründungen von Akademikern nach zeitweiliger Beschäftigung in der Industrie oder in anderen Bereichen der Wirtschaft und Verwaltung.

Zudem bauen große Industrieunternehmen Forschungsbereiche ab und schaffen sich neue Kooperationsnetze 
für die aus Forschungsabteilungen ausgegründeten akademischen Unternehmen. Zum anderen verschmelzen direkt aus Hochschulen und Forschungszentren gegründete akademische Unternehmen oft nach einigen Jahren mit anderen Unternehmen oder gehen in anderen Industrieunternehmen auf. Diesen Kreislauf verdeutlicht die folgende Abbildung:

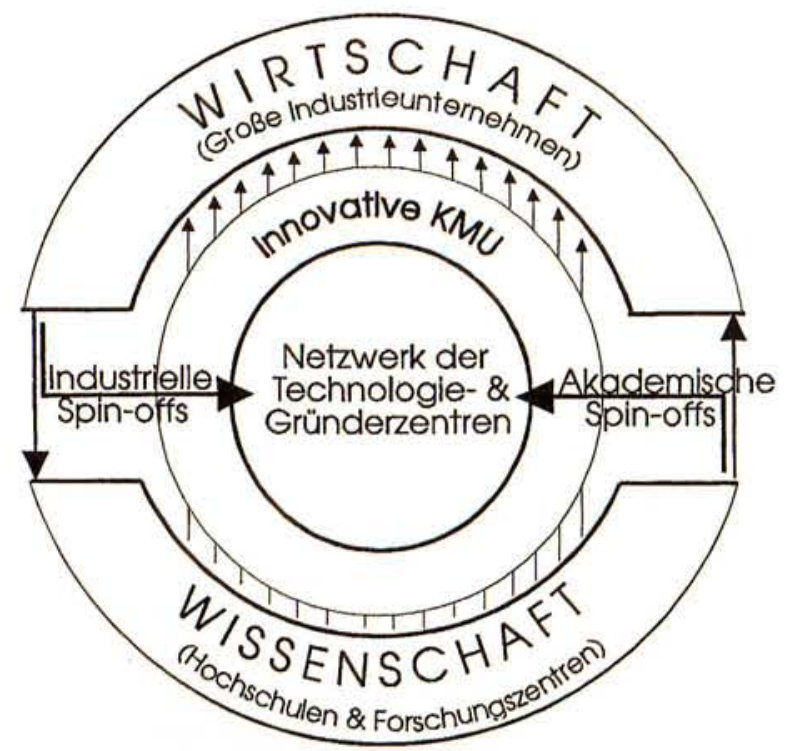

Technologietransfer durch Spin-off-Unternehmensgründungen

Zweifelsohne sind jedoch Unternehmensausgründungen aus Hochschulen und außeruniversitären Forschungseinrichtungen von besonderem Interesse, da es sich hierbei weniger um derivative Spin-offs, sondern um originäre Unternehmensgründungen handelt.

Bildung und Forschung sind die wichtigsten Rohstoffe, über die Deutschland zum Ausgang des 20. Jahrhunderts verfügt und denen es maßgeblich seine Wettbewerbsfähigkeit und Stellung unter den fuihrenden Industrienationen verdankt. Im Rahmen des Projektes ATHENE wurden Unternehmensgründungen aus Hochschulen und Forschungseinrichtungen im technologieorientierten Bereich untersucht. Der beschleunigte Transfer von Forschungsergebnissen in marktfähige Produkte ist das entscheidende Merkmal dieser besonderen Gruppe von Unternehmen.

Die Gründung von Unternehmen durch Akademiker hat in Deutschland Tradition, die durch eine Reihe von heutigen Großunternehmen belegt wird (Siemens, AEG, Hoechst oder - in jüngerer Zeit - SAP). Die derzeitige dynamische Entwicklung der verstärkten wirtschaftlichen Globalisierung führt dazu, daß Forschungsergebnisse zuigiger in marktfähige Produkte und Dienstleistungen umzusetzen sind.

Eine scharfe Trennung zwischen den direkt aus der Wissenschaft erfolgenden Gründungen und den industriellen Spin-offs läßt sich u. E. nicht ziehen, zumal in nicht wenigen Fällen die an der Gründung beteiligten Personen zugleich aus beiden Bereichen kommen. Dennoch reicht der gegenwärtige Stand zur Ausschöpfung der vorhandenen beachtlichen Potentiale nicht aus. Die verstärkten Aktivitäten zur Stimulierung von Ausgründungen können genutzt werden, um die Gründungsraten signifikant zu steigern, ohne daß dies von den betreffenden Einrichtungen als Verlust empfunden und konterkariert wird.

Innovative, technologieorientierte bzw. wissensintensive Unternehmensgründungen stellen nur einen Bruchteil dieser Entwicklung dar, wenngleich ihnen eine strategische volkswirtschaftliche Bedeutung zukommt. Die Mehrzahl der innovativen bzw. technologieorientierten Unternehmen gehört zum F\&E-intensiven verarbeitenden Gewerbe. Nach einem Rückgang der Gründungen in diesem Wirtschaftsbereich in der ersten Hälfte der 90er Jahre kann in Deutschland seit 1995 wieder eine Zunahme festgestellt werden.

Technologie- und Gründerzentren (Gründung des ersten deutschen 1983 in Berlin, heute mehr als 200) leisten einen wichtigen Beitrag bei der Stimulierung und Unterstützung von technologieorientierten Unternehmensgründungen.

Viele der neu gegründeten Technologieunternehmen entwickeln sich schnell. Sie schaffen zum Zeitpunkt der Gründung durchschnittlich 3-4 Arbeitsplätze. Dafür benötigen sie einen Kapitalstock in Höhe von 115.000 DM. Innerhalb von 5 Jahren steigt die Zahl der Beschäftigten auf 10 an. Nach sieben Jahren beschäftigten die Unternehmen im Durchschnitt 12 Mitarbeiter. Damit leisten diese neu entstandenen Unternehmen einen wirksamen Beitrag zur Schaffung von Arbeitsplätzen.

Die Untersuchungen zeigen, daß die jungen technologieorientierten Ausgründungen (mit einem Durchschnittsalter von 35 Jahren) bereits zu $25 \%$ auch auf internationalen Märkten aktiv sind und dort im Mittel $18 \%$ des gesamten Umsatzes realisieren. Dies verdeutlicht, daß hier viele international wettbewerbsfähige Unternehmen entstanden sind.

Die erfolgreichen Gründer mit Hochschulabschluß auf ingenieur- oder naturwissenschaftlichem Gebiet sind in der Regel zwischen 30 und 45 Jahre alt. Bei $63 \%$ der Gründungen hätten fehlende Förderprogramme das Vorhaben verhindert, verzögert oder erschwert. Damit wird deutlich, welch immense Bedeutung gerade dieser Punkt hat.

Die Gründer sind mit dem bisher Erreichten und den Perspektiven sehr zufrieden. Mehr als $83 \%$ der Gründer würden wieder ein Unternehmen gründen. $76 \%$ der Gründer bewerten die bisherige Entwicklung ihres Unternehmens als gut oder sehr gut; 88 schätzen die $\mathrm{Zu}$ kunftsaussichten ihres Unternehmens als gut oder sehr gut ein. Haupttriebfeder waren der Wunsch nach Unabhängigkeit und Selbständigkeit sowie die Verwirklichung eigener Produktideen. Bessere Einkommensmöglichkeiten spielen allerdings nur eine untergeordnete Rolle.

Fehlendes Startkapital $(82 \%)$, zu hohes persönliches Risiko (78\%) oder mangelnde kaufmännische Kenntnisse (75\%) sind die wesentlichen Hemmnisse für die befragten Studenten und Wissenschaftler an Hochschulen, welche sich schon einmal mit dem Gedanken an eine Unternehmensgründung befaßt haben. 


\section{Der Unternehmensbestand im technologie- orientierten Bereich hat sich verdreifacht}

In Deutschland zeichnet sich erfreulicherweise eine neue Gründungswelle ab. Im Jahr 1996 waren - auf der Basis der Gewerbeanmeldungen sowie von Schätzungen des Instituts für Mittelstandsforschung Bonn - insgesamt 502.000 Gründungen zu verzeichnen, denen jedoch zugleich 431.000 Liqudationen gegenuiber standen. 89.400 Handelsregistereintragungen wurden vorgenommen.

Technologieorientierte Unternehmensgründungen haben seit 1990 eine starke Dynamik erlebt. Die Anzahl bestehender Unternehmen hat sich von 1990 bis heute nahezu verdreifacht. Jedes vierte Unternehmen entstand in den NBL. Jedes zweite in den Technologie- und Gründerzentren angesiedelte Unternehmen ist eine Ausgründung aus Wissenschaftseinrichtungen. 1996 befanden sich dort schätzungsweise 2.400 derartige Unternehmen. In den Technologie- und Gründerzentren siedeln sich etwa $33 \%$ der Ausgründungen technologieorientierter Unternehmen an.

Die jährliche Zahl der Gründungen hat sich von $656 \mathrm{im}$ Jahre 1990 auf 1.245 Gründungen im Jahr 1997 nahezu verdoppelt. Dabei schlägt auch eine Gründungswelle in den NBL (und Berlin) zu Buche. Für Unternehmensgründungen aus außeruniversitären Forschungseinrichtungen ergibt sich im Jahre 1997 eine Größenordnung von 150; für Unternehmensgründungen aus Hochschulen (Mitarbeiter und Absolventen/Studenten) ergibt sich im Jahre 1997 schätzungsweise eine Größenordnung von 650. Etwa 450 Unternehmen entstanden durch Gruindungen aus der Industrie. Dennoch ist das hier schlummernde Potential bei weitem noch nicht ausgeschöpft.

\section{Die Rahmenbedingungen für Unternehmens- gründungen in Deutschland sind gut, aber sie sind nicht gut genug.}

Die sich abzeichnende neue Gründungswelle wird durch vielfältige Aktivitäten gefördert. Dazu gehören Existenzgründerseminare an den Hochschulen, Gründertage für Berlin/Brandenburg jährlich im September an der TU Berlin - und Gründermessen in vielen Städten und Regionen, regionale und bundesweite Business-Plan-Wettbewerbe sowie der Einsatz praxiserfahrener Hochschullehrer als Gründungs- und Firmenberater (Coaching). Weitere Beispiele hierfür sind die Gründungsoffensive GO in Nordrhein-Westfalen, das Programm „Offensive Zukunft Bayern“, das Programm „Junge Innovatoren in Baden-Wïrttemberg“, das Projekt „PUG - Prozeßbegleitende Unternehmensgründungen" in Berlin-Brandenburg oder die gemeinsame Berlin/Brandenburger Innovations- und Gründungsoffensive - B INGO u. v. a. m. Speziell auf die Förderung technologieorientierter Unternehmensgründungen und von Existenzgründungen durch Akademiker zielen die Programme „BTU - Beteiligungskapital für kleine Technologieunternehmen“ sowie „FUTOUR - Förderung und Unterstiitzung von technologieorientierten Unternehmensgründungen in den neuen Bundesländern und Berlin (Ost)“ des BMBF, der aktuelle Ideenwettbewerb „EXIST Existenzgründer aus Hochschulen" des BMBF oder solche Landesprogramme wie PFAU in NRW, FLÜGGE in Bayern u. a. Deutschland verfügt über eine Vielzahl von Förderprogrammen. Diese sollten jedoch effizienter und unbürokratischer gestaltet werden.

Akademische Unternehmensgründer brauchen viel Kapital zur Umsetzung ihrer Ideen. In Deutschland muß es gelingen, mehr privates Kapital zu mobilisieren. Die Bereitstellung von Risikokapital muß entsprechend stimuliert werden. Über Beteiligungsgesellschaften hinaus braucht Deutschland mehr „Business Angels“, wie in den USA, als Paten für diese Unternehmungen. Neue Finanzierungsmöglichkeiten sind vor allem für Unternehmensentwicklungen mit mäßigem Wachstum zu erschließen. Um das vorhandene Potential zu erschließen, wurde bereits einiges auf den Weg gebracht. Neue Instrumente bedürfen einer zügigen Umsetzung. Mit dem 3. Finanzmarktförderungsgesetz sowie dem Kapitalaufnahmeerleichterungsgesetz werden sich 1998 die Bedingungen für die Aufnahme von Wagniskapital und die Attraktivität des deutschen Kapitalmarktes erheblich verbessern.

Menschen brauche Beispiele, Gründer brauchen Vorbilder. Zu dem Kreis vieler schon praktizierter und nachahmenswerter Praxis-Beispiele zählen:

- Regionale und bundesweite Business-Plan-Wettbewerbe sowie Innovationspreise

- Intensivierung der Zusammenarbeit zwischen Hochschulen, Forschungszentren, Technologie- und Gründerzentren und der regionalen Wirtschaft

- Starker Anwendungsbezug von Forschung und Lehre sowie breite Einbeziehung in unterschiedliche Tätigkeitsfelder der Auftragsbearbeitung und Realisierung von Forschungsaufträgen

- Möglichkeiten der Nutzung von Einrichtungen und Forschungsgeräten sowie Erteilung von Nutzungsrechten für Patente und Vergabe von Lizenzen

- Praxisorientierte Trainingskurse für Studenten und Mitarbeiter an Hochschulen sowie Forschungseinrichtungen

- Personalkostenzuschüsse für Gründungs- und Aufbauphase eines Unternehmens sowie Schaffung organisatorischer Übergangsregelungen

- Schaffung von Finazierungsfonds für technologieorientierte Unternehmensgründungen aus Hochschulen und Forschungseinrichtungen und deren Wachstum, besonders in Form von „public-private partnerships“

\section{Die Zahl technologieorientierter Unter- nehmensgründungen wächst weiter.}

Nur wenn es gelingt, die in Hochschulen, außeruniversitären Forschungseinrichtungen und den FuE- Abteilungen von Wirtschaftsunternehmen geborenen Ideen rasch in hochwertige Produkte und Dienstleistungen umzusetzen, kann Deutschland sich im internationalen Innovationswettlauf behaupten.

Auf der Basis des vorhandenen Potentials ist davon auszugehen, daß sich der Bestand technologieorientierter 
Unternehmen von 1990 bis zum Jahr 2001 nahezu vervierfacht wird. Durch Kumulation der jährlichen Unternehmensgründungen aus Wissenschaftseinrichtungen im technologieorientierten Bereich, abzüglich der Konkurse und Betriebsaufgaben der entstandenen Unternehmen, im Zeitraum seit 1983 kann der Gesamtbestand im Jahre 2001 auf etwa 14.000 akademische und technologieorientierte Unternehmen abgeschätzt werden.

Die jährliche Zahl der Gründungen erreicht ausgehend von 1.245 Gründungen im Jahr 1997 einen Wert von 1600. Im Zeitraum von 1990 bis 2001 erhöhen sich die Gründungszahlen aus außeruniversitären Forschungseinrichtungen um einen Faktor 2 bis 3 auf ca. 190 im Jahre 2001. Die Unternehmensgründungen aus Hochschulen (Mitarbeiter und Absolventen/Studenten) werden sich mindestens auf ca. 850 bis zum Jahr 2000 erhöhen. Bis zum Jahr 2001 steigen die Gründungen aus der Industrie auf ca. 560 an.

Im Zeitraum von 1990 bis 2001 verdoppelt sich etwa die Zahl der jährlichen Ausgründungen durch Hochschulmitarbeiter. Eine besondere Dynamik zeichnet sich bei Gründungen durch Absolventen und Studenten ab. Im gleichen Zeitraum steigen die Gründungszahlen dieser Personengruppe auf das 2- bis 3-fache.

Die vorliegenden Untersuchungsergebnisse zeigen die hohe Dynamik der Ausgründung technologieorientierter Unternehmen aus Hochschul- und Forschungseinrichtungen. Um eine derartig dynamische Entwicklung auch in Zukunft feststellen zu können, ist es notwendig, das hohe Gründerpotential an deutschen Hochschule und Forschungseinrichtungen verstärkt zu mobilisieren.

\section{Beispiele akademischer Unternehmensent- wicklungen in deutschen Technologie- und Gründerzentren}

Das erste deutsche Technologie- und Gründerzentrum - Berliner Innovations- und Gründerzentrum (BIG) entstand 1983 in Berlin-Wedding mit Gründern aus der TU Berlin. Kurz darauf folgten weitere Technologiezentren (TZ) an Hochschulstandorten (z. B. Aachen, Bremen, Dortmund, Hannover, Heidelberg, Karlsruhe, Konstanz, Ulm). Gleich nach der Wiedervereinigung wurden auch in den neuen Bundesländern und Berlin-Ost die ersten Zentren aufgebaut, meist in der Nähe von Hochschulen oder Forschungseinrichtungen. Nach Angaben der vorliegenden Studie sind in den 200 deutschen Technologie-, Gründer- und Innovationszentren mehr als 5.000 Unternehmen angesiedelt, von denen etwa dreiviertel technologieorientiert sind. Der Beschäftigtenanteil der Akademiker bei den Gesamtbeschäftigten der Zentren liegt bei 50 bis $60 \%$. Im folgenden in Stichpunkten einige Beispiele von TGZ-Unternehmen, die sich in den letzten Jahren besonders erfolgreich entwickelt haben (nach Angaben des ATHENE-Berichts):

AnalytiCon AG (www.analyticon-ag.com) Gegründet 1985 im BIG, heute führend in den Bereichen Biotechnologie und Pharmazie, speziell Qualitätskontrolle biochemischer Forschungen und Entwicklungen, 150 Beschäftigte, seit 1996 an der Börse.

\section{AVM Computersysteme Vertriebs GmbH \& Co.KG \\ (www.avm.de)}

Gehört zu den ersten Unternehmensgründungen im BIG (Berliner Innovations- und Gründerzentrum BerlinWedding), heute fuihrend in der Kommunikationstechnik (ISDN-Controller und Anwendungsprogramme für PC, z. B. 1997 die bekannten FRITZ! Cards zum Anschluß analoger Endgeräte an ISDN), 1998 Umsatz 170 Mio. DM, 220 Mitarbeiter, NL in Frankfurt/M., München und den USA.

Baseler Vision Technologies (www.baslerweb.com) Gegründet 1988 von N. Baseler aus der Hochschule im Technik-Zentrum Luibeck, spezialisiert auf sehende Maschinen, gehört in diesem Segment der Qualitätssicherung zu den Marktführern, heute 110 Beschäftigte, Niederlassungen in den USA, UK und Fernost, 1997 Umsatz 30 Mio. DM.

BioteCon Gesellschaft für Biotechnologische Entwicklung und Consulting $\mathrm{mbH}$ (www.biotecon.com) Gegründet 1990, Tochter von AnalytiCon, 45 Beschäftigte (1998).

\section{CYBERTRON Gesellschaft für Elektronik und Prozeß-} automation mbH (www.cybertron.de)

Gegründet 1983 von H. Klatt aus der TU Berlin im BIG, tätig auf dem Gebiet der Industrieautomation (Robotersysteme, Dosiertechnik, Software-Entwicklung für Qualitätssicherungs-, Fertigungsplanungs- und Steuerungsaufgaben).

\section{ComConsult Kommunikationstechnik GmbH (www.comconsult.de)}

Gegründet 1986 von J. Suppan, einem international anerkannten Netzwerk-Experten, Start 1987 im TZ Aachen, Niederlassungen in Bern, Hamburg und München, heute 140 Mitarbeiter, Gesamtumsatz (1997) 20 Mio. DM, Angebot technologisch hochstehender, herstellerneutraler Dienstleistungen für Netzwerk- und Client-ServerTechnologien, Stammkunden sind die Top 500 der deutschen Industrie.

\section{EUnet Deutschland GmbH/UUNET Deutschland GmbH} (www.uunet.de)

Gegrüindet 1992, seit 1993 im TZ Dortmund, heute 122 Mitarbeiter, europaweite Telekommunikationsleistungen (Internet-Anbindungen), seit 1996 Teil des internationalen Unternehmensverbundes UUNET.

GeSiM mbH (www.gesim.de)

Gegründet als Spin-off aus dem Forschungszentrum Rossendorf (b. Dresden), im ROTECH Rossendorfer Technologiezentrum, Biotechnologie und Genforschung, Innovationspreis der Deutschen Wirtschaft 1998.

IDS Prof. Scheer GmbH (www.ids-scheer.de) Gegrüindet 1985 von Prof. Scheer (Direktor des Instituts für Wirtschaftsinformatik an der Universität des Saarlandes), im SITZ Saarbrücker Innovations- und Technologiezentrum, Softwareentwicklung, weltweit 570 Mitarbeiter, strategischer Partner von SAP, Börsengang 1998 geplant. 
ITI Gesellschaft für ingenieurtechnische Informationsverarbeitung mbH (www.iti.de)

Gegründet 1990, seit 1992 im TZ Dresden, SoftwareTools für Ingenieure (objektorientierte Modellbeschreibung und -lösung), einer der Gründer, Prof. Großmann, ist Leiter des Instituts für Werkzeugmaschinen und Steuerungstechnik an der TU Dresden.

KryptoKom - Gesellschaft für kryptographische Informationssicherheit und Kommunikationstechnologie mbH (www.kryptokom.de)

Gegründet 1988 aus der FH Aachen (Institut für Telematik), im TZ Aachen, marktführendes System- und Softwarehaus für kryptographische Informationssicherheit, 60 Mitarbeiter.

\section{microParts Gesellschaft für Mikrostrukturtechnik mbH (www.microparts.de)}

Gegründet 1990, akademische Spin-off-Gründung unter Beteiligung von 5 großen deutschen Aktiengesellschaften, 25 Patente wurden überlassen, Entwicklung, Produktion und Marketing für die Mikrostrukturtechnik, seit 1994 im Mikrostrukturzentrum NRW im TZ Dortmund, Umsatz 1997 ca. 20 Mio.DM, 1998 Erhöhung auf 32 Mio.DM angestrebt, 80 Mitarbeiter.

Open Soft GmbH (www.tcc-chemnitz.de)

Gegr. 1993 im TZ Chemnitz, Softwarehaus für Automatisierungs-, Informations- und Kommunikationstechnik.

OPTUM Umwelttechnik GmbH (www.tcc-chemnitz.de) Gegründet 1992 im TZ Chemnitz, moderne EDV-Systeme zur Optimierung umwelttechnischer Prozesse (z. B. Wirtschaftlichkeit von Abwasserreinigungsanlagen).

ORBIS - Gesellschaft für Organisation, Beratung, innovatives Systemengineering mbH (www.orbis.de) Gegründet 1986 von Studenten und Professoren der FHTW Saarbrüicken, im SITZ Saarbrüicken, 1997140 Mitarbeiter, Umsatz 18 Mio. DM, Niederlassungen in Hamburg, Paris und Houston (USA).

\section{PARSYTEC Gesellschaft für Parallele Systemtechnik} mbH (www.parsytec.de)

Gegründet 1985 im TZ Aachen, weltweit erster Hersteller von parallelverarbeitenden Industrierechnern auf der Basis neuartiger Transputerchips, 1997 Umsatz 40 Mio. DM, Niederlassungen bzw. Tochterfirmen in Aachen, Chemnitz und Chicago, insgesamt 130 Mitarbeiter.

Pipetronix GmbH (www.pipetronix.de) Gegründet 1983, 1985 bis 1989 in der Technologiefabrik Karlsruhe, Entwicklung und Produktion roboterähnlicher Werkzeuge zum Prüfen von Erdöl- und Gasleitungen , 560 Mitarbeiter, Umsatz 360 Mio. DM.

\section{Sentronic GmbH - Gesellschaft für optische Meß-} systeme (www.sentronic.de)

Gegründet 1993, im TZ Dresden, Außenstelle im TZ Dortmund, moderne optische Online-Meßtechnik (Entwicklung und Produktion von Faseroptiken und Applikationen für Miniaturspektrometer).

\section{Quelle}

Diese Publikation wurde aus Teilen des Abschlußberichts zum Projekt ATHENE - Ausgründungen technologieorientierter Unternehmen aus Hochschulen und außeruniversitären Forschungseinrichtungen - zusammengestellt und redaktionell bearbeitet.

Die Studie war im Auftrag des Bundesministerums für Bildung, Wissenschaft, Forschung und Technologie (BMBF) im Rahmen des Projektes ATHENE von ADTArbeitsgemeinschaft Deutscher Technologie- und Gründerzentren e.V., Berlin (Federführung), FAB -Forschungsagentur Berlin $\mathrm{GmbH}$, Neuenhagen, iAi - Institut für Angewandte Innovationsforschung e.V., Bochum, und bifego - Betriebswirtschaftliches Institut für empirische Gründungs- und Organisationsforschung e.V., Dortmund, erarbeitet worden.

Der vollständige Bericht kann bei der Geschäftsstelle der ADT angefordert werden (198 Seiten; Preis: 232,- DM).

\section{Verfasser}

\author{
Dr. Eberhard Brink \\ Geschäftsführer \\ Technologie- und Gründerzentrum Wildau GmbH \\ Bahnhofstraße, 15745 Wildau \\ Tel. (0 33 75) 508-294
}

\section{Dr. Bernd Groß}

ADT - Arbeitsgemeinschaft Deutscher Technologiezentren e. V. im IGZ Adlershof Rudower Chaussee 5, 12489 Berlin

Tel. (0 30) 63926221 